Artículos Científicos

\title{
Nivel educativo y ocupación de los padres: Su influencia en el rendimiento académico de estudiantes universitarios
}

\author{
Educational Level and Occupation of Parents: His Influence on the \\ Academic Performance of University Students
}

\section{Nível educacional e ocupação parental: sua influência no desempenho acadêmico de estudantes universitários}

\author{
María Vianey Espejel García \\ Instituto Politécnico Nacional, México \\ vianey.espejel@gmail.com \\ https://orcid.org/0000-0001-7307-9379 \\ Martha Jiménez García \\ Instituto Politécnico Nacional, México \\ majimenez@ipn.mx \\ https://orcid.org/0000-0002-8556-2955
}

\section{Resumen}

El rendimiento académico de estudiantes de diferentes niveles educativos ha sido ampliamente analizado considerando factores económicos, sociales o psicológicos. Desde el punto de vista económico, la ocupación de los padres, así como su nivel educativo, muestra una relación positiva con el rendimiento académico de los hijos en diferentes niveles educativos. El objetivo de la presente investigación fue analizar la influencia del nivel educativo y la ocupación de los padres en el rendimiento académico de los estudiantes universitarios y así proponer estrategias que permitan elevar el logro académico. Los datos fueron obtenidos mediante un cuestionario semiestructurado y analizados con el algoritmo chi-squared automatic interaction detection (Chaid), tablas de contingencia y una regresión ordinal. Los resultados muestran que el nivel educativo 


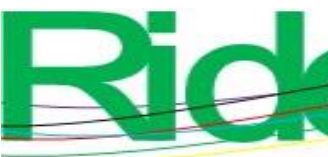

Revista Iberoamericana para la Investigación y el Desarrollo Educativo ISSN $2007-7467$

de la madre, cuando es superior a licenciatura, influye positivamente en el rendimiento académico de los hijos; en cambio el nivel educativo del padre no resultó significativo estadísticamente. Se observó también que estudiantes cuyos padres se desempeñan como profesionistas, comerciantes, directivos, jefes de departamento y trabajadores de la educación son aquellos que obtienen un mejor rendimiento académico; la ocupación de la madre no resultó estadísticamente significativa.

Palabras clave: Chaid, estudiantes, ocupación, padres, rendimiento.

\section{Abstract}

The academic performance of students of different educational levels has been widely analyzed considering from economic, social or psychological factors. From the economic point of view, the occupation of the parents, as well as their educational level, shows a positive relationship with the academic performance of the children in different educational levels. The objective of the present investigation was to analyze the influence of the educational level and the occupation of the parents in the academic performance of the university students and thus propose strategies that allow to elevate the academic achievement. The data were obtained through a semi-structured questionnaire and analyzed with the chi-squared automatic interaction detection (CHAID) algorithm, contingency tables, and an ordinal regression. The results show that the educational level of the mother when she is superior to a truncated degree has a positive influence on the academic performance of the children, whereas the educational level of the father was not statistically significant. It was also observed that students whose parents perform as professionals, traders, managers, department heads, education workers are those who obtain a better academic performance; the occupation of the mother was not statistically significant.

Keywords: CHAID, students, occupation, parents, performance.

\section{Resumo}

O desempenho acadêmico de estudantes de diferentes níveis educacionais tem sido amplamente analisado, considerando fatores econômicos, sociais ou psicológicos. Do ponto de vista econômico, a ocupação dos pais e o nível educacional mostram uma relação positiva com o desempenho acadêmico das crianças nos diferentes níveis de escolaridade. O objetivo da presente investigação foi analisar a influência do nível educacional e a ocupação dos pais no desempenho acadêmico dos estudantes universitários e, assim, 


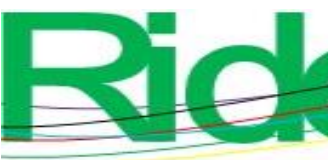

Revista Iberoamericana para la

Investigación y el Desarrollo Educativo

ISSN $2007-7467$

propor estratégias que permitam aumentar o desempenho acadêmico. Os dados foram obtidos através de um questionário semiestruturado e analisados com o algoritmo de detecção automática de interação qui-quadrado (Chaid), tabelas de contingência e regressão ordinal. Os resultados mostram que o nível educacional da mãe, quando superior a um diploma de bacharel, influencia positivamente o desempenho acadêmico dos filhos; Por outro lado, a escolaridade do pai não foi estatisticamente significante. Também foi observado que os alunos cujos pais trabalham como profissionais, comerciantes, gerentes, chefes de departamento e trabalhadores da educação são os que obtêm melhor desempenho acadêmico; a ocupação da mãe não foi estatisticamente significante.

Palavras-chave: Chaid, estudantes, ocupação, pais, atuação.

Fecha Recepción: Junio 2019

Fecha Aceptación: Octubre 2019

\section{Introducción}

El rendimiento académico de estudiantes de diferentes niveles educativos ha sido ampliamente analizado considerando desde factores económicos hasta sociales o psicológicos. El rendimiento académico se refiere al nivel demostrado de conocimientos en un área o materia reflejado a través de indicadores cuantitativos que generalmente están expresados con calificaciones ponderadas en el sistema vigesimal, cuyos rangos de aprobación son establecidos por un grupo social calificado para áreas del conocimiento determinadas (Coschiza, Fernández, Redcozub, Nievas y Ruiz, 2016).

El promedio de calificaciones son el reflejo de evaluaciones y exámenes en los que el estudiante demuestra los conocimientos relacionados con diferentes áreas o materias que se consideran necesarias para su desarrollo como miembro de la sociedad, por lo cual puede ser utilizado como criterio de desempeño académico (Reyes, Godínez, Ariza, Sánchez y Torreblanca, 2014).

En el mismo sentido, Ravelo (2013) indica que el rendimiento académico es resultado de la interacción de múltiples aspectos de lo que le pasa al estudiante de educación superior y se puede medir con las calificaciones obtenidas durante el proceso educativo.

Mamiseishvili (2012) indica que los factores que ayudan a elevar el rendimiento académico son las habilidades para el estudio y la administración del tiempo, el apoyo de la familia, los compañeros y los profesores; es decir, depende de la interacción de diversos 


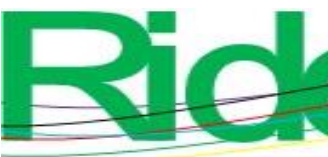

Revista Iberoamericana para la Investigación y el Desarrollo Educativo ISSN $2007-7467$

factores que se relacionan entre sí, tal y como los factores socioeconómicos del entorno familiar, y no solo del sistema escolar (García, Soto y Ochoa, 2013). Cabe mencionar que el apoyo de la familia puede darse de forma monetaria, psicológica, disponibilidad de materiales para el estudio y transferencia de conocimiento. El rendimiento académico está directamente relacionado con el capital humano, considerado un elemento primordial para el crecimiento económico de un país (Baumann y Hamin, 2011).

\section{Ocupación de los padres}

Diversos autores han encontrado que el rendimiento de los alumnos en diferentes niveles académicos tiene relaciones positivas con el nivel socioeconómico de los padres, considerando principalmente la ocupación del padre, debido a que la participación de la mujer en el mercado laboral es baja a pesar de estar en un punto de inflexión. En países desarrollados se ha encontrado que estudiantes cuyos padres son profesionistas o trabajadores de la educación obtienen un rendimiento académico más alto; en cambio, estudiantes cuyos padres están relacionados con el ámbito agrícola tienen un rendimiento académico promedio, $\mathrm{y}$ en países en desarrollo dichos escolares tienden a tener un rendimiento inferior al promedio nacional (Marks, 2011; Chi, Wang, Liu y Zhu, 2017).

Estudiantes cuyos padres tienen ocupaciones básicas o poco cualificadas obtienen un rendimiento menor y son afectados por el riesgo académico con una probabilidad entre $70 \%$ y $80 \%$, lo que muestra de forma clara la diferenciación social (Muelle, 2016). Sin embargo, Coschiza et al. (2016) refieren que la ocupación de los padres no es representativa estadísticamente como una variable de influencia en el rendimiento académico de los estudiantes.

En cuanto al nivel socioeconómico relacionado con la ocupación de los padres, Albor, Dau y Ruíz (2014) refieren que alumnos pertenecientes a niveles socioeconómicos más altos tienen un rendimiento académico superior en licenciaturas como administración, contaduría y medicina. El bajo rendimiento académico en los niveles socioeconómicos bajos se debe a que este impide a los estudiantes el acceso a fuentes y recursos de aprendizaje como tecnología, cursos intensivos, etc. (Reyes et al., 2014). 


\section{Nivel de estudio de los padres}

El nivel educativo de los padres es considerado un componente central en el capital cultural de los estudiantes, ya que determina las habilidades, valores y conocimientos de estos con respecto a la educación formal y en sus prácticas educativas, además de incrementar sus habilidades verbales, cognitivas y espaciales; lo anterior tiene una relación directa con el rendimiento académico del estudiante (Díaz y Morales, 2011; Chaparro, González y Caso, 2016).

Tal como indican Rodríguez y Guzmán (2019), el nivel educativo de los padres es identificado como uno de los factores familiares que inciden en el rendimiento académico de los estudiantes, y que estudiantes con padres con estudios universitarios tienden a tener un mejor desempeño escolar. La educación de los padres mejora la manera en que interactúan los miembros de la familia al fomentar la adopción de guiones cognitivos, creencias y valores que están relacionados con el comportamiento académico y se relaciona con la consecución de logros (Abuya, Mumah, Austrian, Mutisya y Kabiru, 2018). El nivel educativo de los padres en promedio ayuda a determinar las expectativas de la participación de sus hijos en niveles superiores de educación (Koshy, Dockery y Seymour, 2019).

Por su parte, Erola, Jalonen y Lehti (2016) mencionan que la influencia del nivel académico de los padres, la ocupación y el ingreso en el rendimiento académico está interrelacionada, ya que cierto tipo de educación conduce a ocupaciones específicas y proporciona ciertos niveles de ingreso; por lo tanto, la acumulación de educación en forma de capital humano en los padres coadyuva no solo a la mejora del nivel socioeconómico familiar, también influye en una mejora del rendimiento académico.

Diversos estudios han encontrado que el nivel educativo de las madres tiene una mayor relevancia en el logro académico de los estudiantes en diferentes niveles académicos; por lo que estudiantes con madres que tienen educación superior son los que obtienen un mejor rendimiento en el aula (Díaz y Morales, 2011; Manchón y Cordero, 2014; Harding, Morris y Hughes, 2015; Chaparro et al, 2016; Waterman y Lefkowitz, 2017; Abuya et al., 2018; Simões, Rivera, Moreno y Gaspar de Matos, 2018; Kim, 2018; De Coninck, Matthijs y Luyten, 2019). En países en desarrollo se considera que la importancia de la educación materna en el rendimiento académico probablemente esté asociada al rol tradicional de la mujer en la formación de los hijos (Cuenca, 2016).

Debido a la complejidad del rendimiento académico como objeto de estudio por los múltiples factores que influyen en el mismo, en la presente investigación se tomó en cuenta únicamente el nivel educativo, la ocupación de los padres y el ingreso económico 


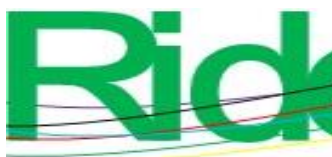

Revista Iberoamericana para la Investigación y el Desarrollo Educativo ISSN 2007 - 7467

familiar. Por lo cual, el objetivo de este estudio fue analizar la influencia de la ocupación y el nivel de estudio de los padres en el rendimiento académico de los estudiantes universitarios y poder identificar estrategias para elevar el logro académico.

La hipótesis de la investigación es que el nivel educativo de la madre y la ocupación del padre tienen influencia positiva en el rendimiento académico de los estudiantes universitarios en México.

\section{Materiales y métodos}

El estudio fue de tipo exploratorio transversal realizado con la finalidad de determinar la influencia del nivel académico y la ocupación de los padres en el rendimiento académico de los estudiantes universitarios. Los datos fueron recopilados en México en el año 2017. La investigación estuvo dirigida a estudiantes universitarios pertenecientes a universidades públicas. Se consideraron como variables independientes la clasificación del nivel de estudios de la madre y del padre (Cla_NEM y Cla_NEP), clasificación del ingreso económico familiar (Clas_ingresos), la ocupación del padre y de la madre según la clasificación mexicana de ocupaciones publicada por el Instituto Nacional de Estadística y Geografía [Inegi] (ClaP_Inegi y ClaM_Inegi); y como variable dependiente se consideró el promedio de calificaciones del estudiante universitario (re_aca), considerada como variable proxy del rendimiento académico. En la tabla 1 se muestra la descripción de las variables. 
Tabla 1. Descripción de variables del modelo

\begin{tabular}{|c|c|c|}
\hline Variable & Descripción & Relación \\
\hline $\begin{array}{l}\text { Rendimiento académico } \\
\text { (re_aca) }\end{array}$ & $\begin{array}{l}\text { Promedio de calificación } \\
\text { obtenida por el estudiante } \\
\text { universitario, considera los } \\
\text { niveles: excelente (9.6-10), } \\
\text { bueno (8.6-9.5), adecuado } \\
(7.6-8.5) \text { y deficiente (6.0- } \\
7.5) \text {. }\end{array}$ & $\begin{array}{l}\text { Dependiente: variable } \\
\text { primordial para analizar la } \\
\text { influencia de la ocupación } \\
\text { de los padres en el } \\
\text { rendimiento académico del } \\
\text { estudiante universitario. }\end{array}$ \\
\hline $\begin{array}{l}\text { Clasificación del nivel de } \\
\text { estudios de la madre } \\
\text { (Cla_NEM) }\end{array}$ & $\begin{array}{l}\text { Nivel de estudios de la } \\
\text { madre del estudiante } \\
\text { universitario; considera } \\
\text { nueve niveles hasta } \\
\text { doctorado incluyendo sin } \\
\text { estudios. }\end{array}$ & $\begin{array}{l}\text { Independiente: variable } \\
\text { considerada por la } \\
\text { influencia que puede tener } \\
\text { en el rendimiento } \\
\text { académico del estudiante } \\
\text { universitario al transferir } \\
\text { conocimientos. }\end{array}$ \\
\hline \begin{tabular}{l}
\multicolumn{3}{l}{ Clasificación del nivel de } \\
estudios del padre \\
(Cla_NEP)
\end{tabular} & $\begin{array}{l}\text { Nivel de estudios del padre } \\
\text { del estudiante } \\
\text { universitario; considera } \\
\text { nueve niveles hasta } \\
\text { doctorado incluyendo sin } \\
\text { estudios. }\end{array}$ & $\begin{array}{l}\text { Independiente: variable } \\
\text { considerada por la } \\
\text { influencia que puede tener } \\
\text { en el rendimiento } \\
\text { académico del estudiante } \\
\text { universitario al transferir } \\
\text { conocimientos. }\end{array}$ \\
\hline $\begin{array}{lr}\text { Clasificación del ingreso } \\
\text { económico } & \text { familiar } \\
\text { (Clas_ingresos) } & \end{array}$ & $\begin{array}{lr}\text { Ingreso } & \text { económico } \\
\text { mensual } & \text { familiar; } \\
\text { contempla cinco niveles de } \\
\text { ingreso. }\end{array}$ & $\begin{array}{l}\text { Independiente: } \\
\text { económica considerada } \\
\text { porque puede proporcionar } \\
\text { medios físicos (libros, } \\
\text { tecnología, etc.) que } \\
\text { influyen en el rendimiento } \\
\text { académico. }\end{array}$ \\
\hline $\begin{array}{l}\text { Ocupación del padre } \\
\text { según clasificación del } \\
\text { Inegi (ClaP_Inegi) }\end{array}$ & $\begin{array}{lrr}\text { Ocupación } & \text { del padre del } \\
\text { estudiante } & \text { universitario } \\
\text { considerando } & \text { la } \\
\text { clasificación } & \text { mexicana de } \\
\text { ocupación } & \text { del } & \text { Inegi; } \\
\text { considera } & 19 & \text { áreas } \\
\text { generales. } & & \\
\end{array}$ & $\begin{array}{l}\text { Independiente: variable } \\
\text { considerada para } \\
\text { determinar su influencia en } \\
\text { el rendimiento académico. }\end{array}$ \\
\hline $\begin{array}{l}\text { Ocupación de la madre } \\
\text { según clasificación del } \\
\text { Inegi (ClaM_Inegi) }\end{array}$ & $\begin{array}{lrr}\text { Ocupación de la madre del } \\
\text { estudiante } & \text { universitario } \\
\text { considerando } & \text { la } \\
\text { clasificación } & \text { mexicana de } \\
\text { ocupación } & \text { del } & \text { Inegi; } \\
\text { considera } & 19 & \text { áreas } \\
\text { generales. } & & \\
\end{array}$ & $\begin{array}{l}\text { Independiente: variable } \\
\text { considerada para } \\
\text { determinar su influencia en } \\
\text { el rendimiento académico. }\end{array}$ \\
\hline
\end{tabular}

Fuente: Elaboración propia

Para la recopilación de datos se diseñó y aplicó una encuesta semiestructurada que incluyó secciones relacionadas con las variables mencionadas anteriormente. La encuesta fue aplicada de forma aleatoria; el tamaño de la muestra fue equivalente a 918 estudiantes 
que se encontraban cursando estudios universitarios. Los datos fueron obtenidos en 2017 en Ciudad de México.

En el estudio se utilizó el método de análisis multivariado llamado en inglés chisquared automatic interaction detection (Chaid), el cual, cabe añadir, es un algoritmo exhaustivo, y el modelo de regresión ordinal. El primero refiere a una técnica de análisis de segmentación que permite establecer relaciones de dependencia entre variables explicadas y explicativas (Escobar, 1992). Para complementar el árbol de clasificación obtenido mediante el algoritmo Chaid exhaustivo se incluyen tablas de contingencia. Por su parte, el modelo de regresión ordinal está diseñado para el análisis de variables respuesta medidas en una escala ordinal (Rodríguez G., 2007).

Los datos fueron capturados y analizados con el paquete estadístico IBM SPSS Statistics 20.0. El modelo de regresión ordinal consideró como variable dependiente el rendimiento académico de los estudiantes universitarios (re_aca). Los predictores considerados fueron: clasificación de nivel de estudios de la madre y del padre (Cla_NEM y Cla_NEP, respectivamente), clasificación del ingreso familiar (Clas_ingresos) y la ocupación del padre y de la madre según la clasificación del Inegi (ClaP_Inegi y ClaM_Inegi, respectivamente).

\section{Resultados y discusión}

Los resultados obtenidos muestran que $60 \%$ de los estudiantes universitarios son hombres y $40 \%$ mujeres. En cuanto a las calificaciones obtenidas, se observa que el promedio es igual a 8 puntos en la escala de 0 a 10. En cuanto al nivel de estudios de los padres, $35.7 \%$ de las madres cuentan con estudios de bachillerato, mientras que $36.9 \%$ de los padres cuentan con estudios de licenciatura. Se encontró que el ingreso promedio se encuentra entre 4611 y 6129 pesos mensuales y que los padres trabajan principalmente en el sector privado.

En la figura 1 se muestra el árbol de clasificación obtenido a través del algoritmo Chaid exhaustivo. 


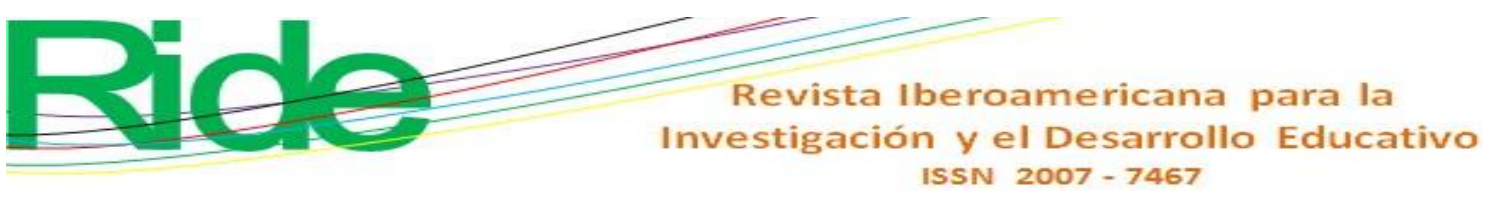

Figura 1. Árbol de clasificación

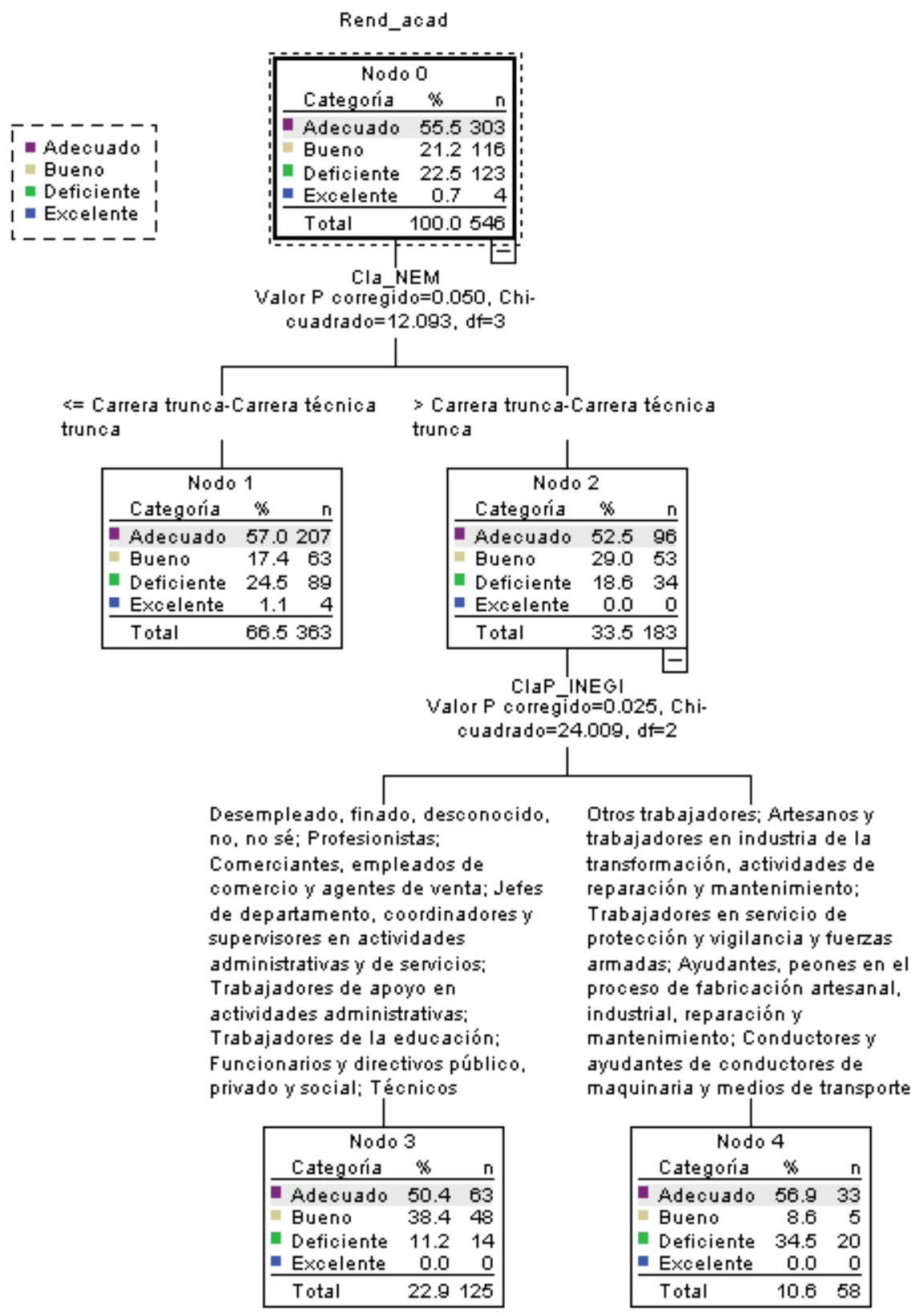

Fuente: Elaboración propia

El árbol de clasificación muestra que, del total de estudiantes universitarios encuestados, solo $0.7 \%$ tiene un rendimiento excelente, es decir, un promedio de calificaciones superior a 9.6; por otra parte, $21.2 \%$ tiene un rendimiento bueno (9.5-8.6); $55.5 \%$ tiene un rendimiento académico adecuado, es decir, entre 7.6 y 8.5 de promedio de calificaciones, y $22.5 \%$ tiene un rendimiento deficiente (7.5-6.0).

Se identificó al nivel de estudios de la madre como la principal variable predictora con una ji al cuadrado de 12.093. Se observa que de los estudiantes cuyas madres tienen educación superior a licenciatura trunca $29.0 \%$ obtuvieron un rendimiento académico 


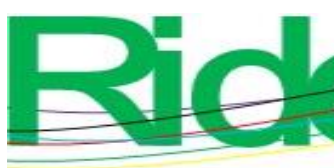

Revista Iberoamericana para la Investigación y el Desarrollo Educativo ISSN $2007-7467$

bueno; sin embargo, ninguno obtuvo un rendimiento académico excelente. Cabe señalar que el porcentaje de estudiantes que obtuvieron un rendimiento académico bueno y cuyas madres tienen un nivel académico menor a la licenciatura fue inferior en casi 12 puntos porcentuales - lo cual concuerda con los estudios realizados por Marks (2011); Díaz y Morales (2011); Manchón y Cordero (2014), y De Coninck et al. (2019). Lo anterior se debe a que principalmente en América Latina las madres están presentes en la mayor parte de la educación de los hijos y al tener un nivel de estudio más alto la capacidad para brindar apoyo efectivo a los escolares es mayor.

La siguiente variable predictora del rendimiento académico es la clasificación de la ocupación del padre según el Inegi. Al respecto, se percibe que el porcentaje de estudiantes con un rendimiento académico bueno (38.4\%) son aquellos cuyos padres son profesionistas, comerciantes, directivos, trabajadores administrativos y de la educación; en cambio los estudiantes cuyos padres están ocupados en la industria de la transformación, mantenimiento y reparación en niveles operativos y que obtuvieron un rendimiento académico bueno representaron solo $8.6 \%$. Esto concuerda con lo reportado por Albor et al. (2014); Muelle (2016); Canales (2016) y Chi et al. (2017). Lo anterior se debe a que empleos con niveles de ingreso más alto permiten a los padres crear ambientes propicios para el desarrollo educativo de sus hijos, al proporcionarles medios físicos, digitales y de seguridad para el desarrollo de sus habilidades, lo que pone en evidencia que aún existe desigualdad en el sistema de educación en México y en otros países latinoamericanos.

Rodríguez y Guzmán (2019) indican que los alumnos cuyos padres se encuentran en una situación laboral con mayor inestabilidad o precariedad son más vulnerables a tener un rendimiento académico bajo y a abandonar la escuela (Yáñez, Vera y Mungarro, 2014; Reyes et al., 2014; Cuenca, 2016; Chaparro et al., 2016; Rodríguez y Guzmán, 2019). Sin embargo, se observa que $56.9 \%$ de los estudiantes cuyos padres se emplean en la industria de transformación, mantenimiento y reparación en niveles operativos tienen un rendimiento académico adecuado; mientras que solo $50.4 \%$ de los estudiantes cuyos padres se emplean como profesionistas, comerciantes, directivos, trabajadores administrativos y de la educación obtienen dicho rendimiento. Lo anterior muestra que, aunque los estudiantes con un nivel académico adecuado son aquellos cuyos padres se ocupan en el nivel operacional, los estudiantes con padres ocupados en niveles directivos y profesionistas son los que obtienen un rendimiento académico más alto. 
En la tabla 2 se muestra el análisis de correlación de las variables nivel de estudios de la madre para determinar su influencia en el rendimiento académico del estudiante universitario.

Tabla 2. Rendimiento académico según clasificación del nivel de estudios de la madre

\begin{tabular}{|c|c|c|c|c|c|c|c|c|c|c|}
\hline \multirow{2}{*}{\multicolumn{2}{|c|}{ Ren_aca }} & \multicolumn{9}{|c|}{ Cla_NEM } \\
\hline & & & \multirow{2}{*}{\begin{tabular}{|l|}
1 \\
30
\end{tabular}} & \multirow{2}{*}{$\begin{array}{l}\mathbf{2} \\
112\end{array}$} & \multirow{2}{*}{\begin{tabular}{|r|}
3 \\
1
\end{tabular}} & \multirow{2}{*}{\begin{tabular}{|l|}
4 \\
196
\end{tabular}} & \multirow{2}{*}{$\begin{array}{r}5 \\
1\end{array}$} & \multirow{2}{*}{$\begin{array}{l}\mathbf{6} \\
151\end{array}$} & \multirow{2}{*}{\begin{tabular}{|l|}
7 \\
13
\end{tabular}} & \multirow{2}{*}{$\begin{array}{r}8 \\
1\end{array}$} \\
\hline \multirow{2}{*}{ Adecuado } & Núm. & & & & & & & & & \\
\hline & $\%$ & 0.1 & 3.3 & 12.2 & 0.1 & 21.4 & 0.1 & 16.5 & 1.4 & 0.1 \\
\hline \multirow{2}{*}{ Bueno } & Núm. & 0 & 17 & 44 & 0 & 61 & 1 & 82 & 8 & 0 \\
\hline & $\%$ & 0.0 & 1.9 & 4.8 & 0.0 & 6.7 & 0.1 & 9.0 & 0.9 & 0.0 \\
\hline \multirow{2}{*}{ Deficiente } & Núm. & 2 & 9 & 55 & 0 & 66 & 0 & 48 & 5 & 5 \\
\hline & $\%$ & 0.2 & 1.0 & 6.0 & 0.0 & 7.2 & 0.0 & 5.2 & 0.5 & 0.5 \\
\hline \multirow{2}{*}{ Excelente } & Núm. & 0 & 0 & 1 & 0 & 4 & 0 & 1 & 0 & 0 \\
\hline & $\%$ & 0.0 & 0.0 & 0.1 & 0.0 & 0.4 & 0.0 & 0.1 & 0.0 & 0.0 \\
\hline \multirow{2}{*}{ Total } & Núm. & 3 & 56 & 212 & 1 & 327 & 2 & 282 & 26 & 6 \\
\hline & $\%$ & 0.3 & 6.1 & 23.2 & 0.1 & 35.7 & 0.2 & 30.8 & 2.8 & 0.7 \\
\hline
\end{tabular}

Fuente: Elaboración propia

Los números de identificación de la tabla 2 son los siguientes. 0: Sin estudios, 1: Primaria, 2: Secundaria, 3: Bachillerato trunco, 4: Bachillerato, 5: Licenciatura trunca, 6: Licenciatura, 7: Maestría, 8: Doctorado.

El valor estadístico $\chi^{2}$ calculados para el rendimiento académico respecto al nivel educativo de la madre es 39.528, la probabilidad de encontrar una $\chi_{c}^{2}$ menor a una $\chi_{t}^{2}$ es baja (0.024) por lo que se rechaza la hipótesis nula en favor de la hipótesis alternativa que establece que la distribución entre las casillas es diferente. Se observa que existe una alta correlación entre el rendimiento académico adecuado del estudiante y el nivel académico de la madre cuando éste es equivalente al nivel bachillerato (21.4\%). Esto concuerda con lo reportado por diversos autores que indican que existe una asociación positiva entre el nivel educativo de las madres y el rendimiento académico, debido a que madres con un nivel educativo más alto o mayor capital humano utilizan prácticas de crianza de mayor calidad para estimular cognitivamente a sus hijos además de participar más en actividades escolares de lo que los padres lo hacen (Harding et al., 2015; Erola, Jalonen y Lehti, 2016; Abuya et al., 2018; Simões et al., 2018; Takeda y Lamichhane, 2018; Kim, 2018). 


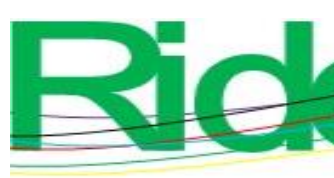

Revista Iberoamericana para la Investigación y el Desarrollo Educativo ISSN $2007-7467$

En la tabla 3 se muestra el análisis de correlación de la variable ocupación del padre para determinar su influencia en el rendimiento académico del estudiante universitario.

Tabla 3. Rendimiento académico según ocupación del padre considerando clasificación del Inegi

\begin{tabular}{|c|c|c|c|c|c|c|c|c|c|c|c|c|c|c|c|c|c|}
\hline \multirow{2}{*}{\multicolumn{2}{|c|}{ Re_aca }} & \multicolumn{16}{|c|}{ ClaP_Inegi } \\
\hline & & \multirow{2}{*}{\begin{tabular}{|l|}
$\mathbf{0}$ \\
20
\end{tabular}} & \multirow{2}{*}{$\begin{array}{l}1 \\
80\end{array}$} & \multirow{2}{*}{$\begin{array}{l}2 \\
10\end{array}$} & 3 & 4 & 5 & 6 & & \multirow{2}{*}{$\begin{array}{c}8 \\
30\end{array}$} & 9 & 10 & & \begin{tabular}{|l}
12 \\
\end{tabular} & \multirow{2}{*}{\begin{tabular}{|r|}
13 \\
2
\end{tabular}} & \multirow{2}{*}{$\begin{array}{r}14 \\
6\end{array}$} & \multirow{2}{*}{$\begin{array}{l}\mathbf{1 5} \\
118\end{array}$} \\
\hline Ade & Núr & & & & 21 & & 1 & & 15 & & & 1 & 114 & 0 & & & \\
\hline & $\%$ & 2.4 & 8.7 & 1.1 & 2.3 & 2.4 & 0.1 & 4.5 & 1.6 & 3.3 & 2.5 & 0.1 & 12.5 & 0.0 & 0.2 & 0.7 & 12.9 \\
\hline \multirow[t]{2}{*}{ Bueno } & a. & 7 & 38 & 0 & 7 & 14 & 2 & 18 & 2 & 10 & 9 & 7 & 65 & 0 & 1 & 1 & 32 \\
\hline & $\%$ & 0.8 & 4.2 & 0.0 & 0.8 & 1.5 & 0.2 & 2.0 & 0.2 & 1.1 & 1.0 & 0.8 & 7.1 & 0.0 & 0.1 & 0.1 & 3.5 \\
\hline \multirow[t]{2}{*}{ Deficiente } & im. & 4 & 20 & 2 & 11 & 9 & 1 & 21 & 4 & 16 & 5 & 5 & 36 & 2 & 1 & 3 & 50 \\
\hline & $\%$ & 0.4 & 2.2 & 0.2 & 1.2 & 1.0 & 0.1 & 2.3 & 0.4 & 1.7 & 0.5 & 0.5 & 3.9 & 0.2 & 0.1 & 0.3 & 5.5 \\
\hline \multirow[t]{2}{*}{ Excelente } & Núm. & 1 & 1 & 0 & 0 & 0 & 0 & 0 & 0 & 0 & 0 & 0 & 0 & 0 & 0 & 0 & 4 \\
\hline & $\%$ & 0.1 & 0.1 & 0.0 & 0.0 & 0.0 & 0.0 & 0.0 & 0.0 & 0.0 & 0.0 & 0.0 & 0.0 & 0.0 & 0.0 & 0.0 & 0.4 \\
\hline \multirow[t]{2}{*}{ Total } & Núm. & 34 & 139 & 12 & 39 & 45 & 4 & 80 & 21 & 56 & 37 & 13 & 215 & 2 & 4 & 10 & 204 \\
\hline & $\%$ & 3.7 & 15.2 & 1.3 & 4.3 & 4.9 & 0.4 & 8.7 & 2.3 & 6.1 & 4.0 & 1.4 & 23.5 & 0.2 & 0.4 & 1.1 & 22.3 \\
\hline
\end{tabular}

Fuente: Elaboración propia

Los números de identificación de la tabla 3 se describen a continuación. 0: Desempleado, finado, desconocido; 1: Profesionista; 2: Técnicos; 3: Trabajadores de la educación; 4: Funcionarios y directivos público, privado y social; 5: Trabajadores agrícolas, ganaderos, silvícolas, caza y pesca; 6: Artesanos y trabajadores de la industria de la transformación, actividades de reparación y mantenimiento; 7: Ayudantes, peones en el proceso de fabricación artesanal, industrial, reparación y mantenimiento; 8: Conductores y ayudantes de conductores de maquinaria y medios de transporte; 9: Jefes de departamento, coordinadores y supervisores en actividades administrativas y de servicios; 10: Trabajadores de apoyo en actividades administrativas; 11: Comerciantes, empleados de comercio y agentes de venta; 12: Vendedores ambulantes y trabajadores ambulantes en servicios; 13: Trabajadores en servicios personales; 14: Trabajadores en servicio de protección, vigilancia y fuerzas armadas, y 15: Otros trabajadores.

El valor estadístico $\chi^{2}$ calculado para el rendimiento académico respecto a la ocupación del padre es de 69.132; la probabilidad de encontrar una $\chi_{c}^{2}$ menor a una $\chi_{t}^{2}$ es baja (0.012), por lo que se rechaza la hipótesis nula en favor de la hipótesis alternativa 
que establece que la distribución entre las casillas es diferente. Se observa que existe una alta correlación entre el rendimiento académico adecuado del estudiante y cuando el padre se emplea como comerciante, empleado de comercio y agente de ventas (12.5\%). Lo anterior concuerda con los hallazgos realizados por Marks (2011); Díaz y Morales (2011); Reyes et al. (2014); Casanova et al. (2018), y Chi et al. (2017), quienes indican que los estudiantes cuyos padres tienen ocupaciones con mejores ingresos suelen tener un mejor rendimiento académico, debido a que los mejores ingresos permiten proveer de más y mejores medios para el desarrollo de habilidades académicas; en cambio, estudiantes provenientes de entornos económicos desfavorecidos pueden tener un rendimiento académico bajo ocasionado por habilidades deficientes y malos hábitos de estudio a raíz de las carencias que pudieran presentar, así como la falta de acceso a fuentes y recursos de aprendizaje.

El segundo análisis se llevó a cabo a través de un modelo de regresión ordinal. A continuación, se muestran las pruebas de ajuste del modelo, la prueba de líneas paralelas y los coeficientes obtenidos. La tabla 4 muestra contiene la información del ajuste del modelo de regresión ordinal y la tabla 5 los datos del pseudo R-cuadrado.

Tabla 4. Información de ajuste del modelo de regresión ordinal

\begin{tabular}{|l|r|r|r|r|}
\hline \multicolumn{1}{|c|}{ Modelo } & $\begin{array}{c}-2 \text { log de la } \\
\text { verosimilitud }\end{array}$ & Chi-cuadrado & \multicolumn{1}{c|}{ gl } & Sig. \\
\hline Solo intersección & 1518.21 & & & \\
\hline Final & 1424.66 & 93.55 & 47 & .000 \\
\hline Función de vínculo: Logit.
\end{tabular}

Fuente: Elaboración propia

Tabla 5. Datos del pseudo R-cuadrado del modelo de regresión ordinal

\begin{tabular}{|l|r|}
\hline Cox y Snell & .097 \\
\hline Nagelkerke & .112 \\
\hline McFadden & .050 \\
\hline \multicolumn{2}{|l|}{ Función de vínculo: Logit. } \\
\hline
\end{tabular}

Fuente: Elaboración propia 
Se considera que el modelo cuenta con un buen ajuste, pues al comparar el nivel de significancia de 0.002 , con un $\alpha=0.05$; se rechaza la hipótesis nula que establece que el modelo sin variables predictivas es mejor que el modelo con variables predictivas. La tabla 6 muestra las estimaciones de los parámetros del modelo de regresión ordinal.

Tabla 6. Estimaciones de los parámetros del modelo de regresión ordinal

\begin{tabular}{|c|c|c|c|c|c|c|c|c|}
\hline & \multirow[t]{2}{*}{ Estimación } & \multirow[t]{2}{*}{$\begin{array}{l}\text { Error } \\
\text { típ. }\end{array}$} & \multirow[t]{2}{*}{ Wald } & \multirow[t]{2}{*}{ gl } & \multirow[t]{2}{*}{ Sig. } & \multicolumn{2}{|c|}{$\begin{array}{c}\text { Intervalo de } \\
\text { confianza } 95 \%\end{array}$} \\
\hline & & & & & & & \begin{tabular}{|c|} 
Límite \\
inferior
\end{tabular} & $\begin{array}{c}\text { Límite } \\
\text { superior }\end{array}$ \\
\hline \multirow{4}{*}{ Umbral } & {$[$ re_aca $=1.00]$} & -8.180 & 1.313 & 38.80 & 1 & .000 & -10.754 & -5.606 \\
\hline & {$[$ re_aca $=2.00]$} & -4.169 & 1.247 & 11.17 & 1 & .001 & -6.613 & -1.724 \\
\hline & {$[$ re_aca $=3.00]$} & -1.484 & 1.243 & 1.43 & 1 & .232 & -3.920 & .952 \\
\hline & [Cla_NEM=1] & -3.346 & 1.240 & 7.28 & 1 & .007 & -5.776 & -.916 \\
\hline \multirow{10}{*}{ Ubicación } & [Cla_NEM=2] & -2.754 & 1.212 & 5.17 & 1 & .023 & -5.129 & -.379 \\
\hline & [Cla_NEM=4] & -2.795 & 1.206 & 5.38 & 1 & .020 & -5.158 & -.432 \\
\hline & [Cla_NEM=5] & -4.240 & 1.839 & 5.31 & 1 & .021 & -7.845 & -.634 \\
\hline & [Cla_NEM=6] & -3.294 & 1.195 & 7.60 & 1 & .006 & -5.637 & -.952 \\
\hline & [Cla_NEM=7] & -3.254 & 1.205 & 7.28 & 1 & .007 & -5.616 & -.891 \\
\hline & [ClaP_Inegi=11] & -.520 & .207 & 6.32 & 1 & .012 & -.925 & -.115 \\
\hline & [Cla_NEP=4] & 2.511 & 1.225 & 4.20 & 1 & .040 & .110 & 4.912 \\
\hline & [ClaM_Inegi=4] & -3.607 & 1.394 & 6.70 & 1 & .010 & -6.338 & -.875 \\
\hline & [ClaM_Inegi=10] & -2.782 & .916 & 9.22 & 1 & .002 & -4.578 & -.987 \\
\hline & [ClaM_Inegi=14] & -.853 & .326 & 6.85 & 1 & .009 & -1.491 & -.214 \\
\hline
\end{tabular}

Fuente: Elaboración propia

Las estimaciones de la regresión están etiquetadas como "umbral" para los términos equivalentes al intercepto y "ubicación" son los coeficientes para la variable predictiva. Al realizar las pruebas de significancia para cada uno de los coeficientes, considerando un $\alpha=0.05$, se observa que el nivel de estudios de la madre (Cla_NEM) y del padre (Cla_NEP), la ocupación del padre y de la madre según la clasificación del Inegi (ClaP_Inegi y ClaM_Inegi), están relacionados con el rendimiento académico de los estudiantes universitarios (re_aca). 


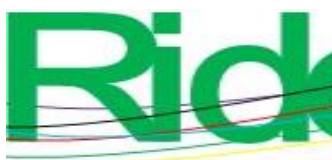

Revista Iberoamericana para la Investigación y el Desarrollo Educativo ISSN $2007-7467$

De manera concreta, se observa que es más probable que el rendimiento académico adecuado y bueno resulte afectado por el nivel de estudios de los padres, principalmente cuando es equivalente a licenciatura trunca en el caso de los padres y en todos los niveles (excepto bachillerato trunco) en el caso de las madres. En cuanto a la ocupación de los padres, se observa que es más probable que niveles de rendimiento adecuado y bueno sean afectados cuando el padre es comerciante o empleado de comercio y agente de ventas; por otro lado, cuando las madres trabajan como ayudantes o peones en la fabricación artesanal e industrial es probable que influyan en el rendimiento académico de sus hijos. La tabla 7 contiene los datos de la prueba de líneas paralelas de la regresión estimada.

Tabla 7. Datos de la prueba de líneas paralelas de la regresión ordinal

\begin{tabular}{|l|r|r|r|r|}
\hline \multicolumn{1}{|c|}{ Modelo } & $\begin{array}{r}\text {-2 log de la } \\
\text { verosimilitud }\end{array}$ & \multicolumn{1}{c|}{$\begin{array}{c}\text { Chi- } \\
\text { cuadrado }\end{array}$} & \multicolumn{1}{c|}{ gl } & \multicolumn{1}{c|}{ Sig. } \\
\hline Hipótesis nula & 1424.66 & & & \\
General & 1314.42 & 110.23 & 94 & .121 \\
\hline
\end{tabular}

Fuente: Elaboración propia

Respecto a la prueba de líneas paralelas, la hipótesis nula establece que los coeficientes de las pendientes son los mismos para todas las categorías de la variable respuesta; por lo cual, al tener un nivel de significancia de 0.121 y considerando un $\alpha=$ 0.05 , no se rechaza la hipótesis nula y se concluye que los coeficientes de las pendientes son iguales para todas las categorías de la variable respuesta.

La presente investigación permite vislumbrar la importancia de la influencia de aspectos socioeconómicos de los padres en el rendimiento académico de los estudiantes universitarios, lo que permitirá determinar la forma en que se diseñan y aplican los incentivos a los estudiantes para mejorar su rendimiento académico. Sin embargo, no hay que olvidar que en la muestra se contemplaron a estudiantes de universidades públicas, sin identificar a alumnos de universidades privadas. La fortaleza de esta investigación consiste en la utilidad para el diseño y aplicación de estrategias que permitan incentivar a los estudiantes universitarios para la mejora de su desempeño académico. 


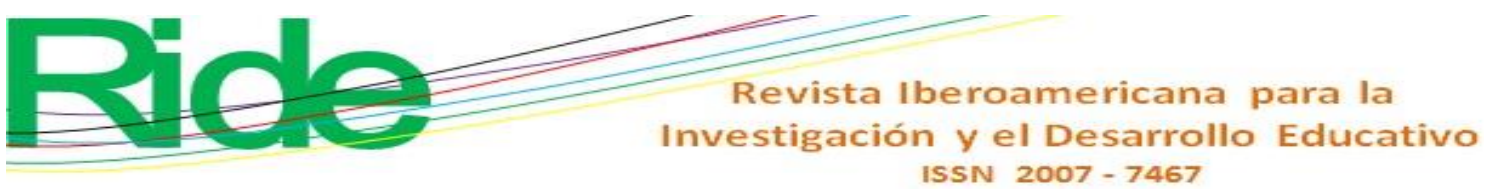

\section{Conclusiones}

Se encontró que el rendimiento académico de los estudiantes universitarios es influenciado principalmente por el nivel de estudios de la madre y la ocupación del padre; lo anterior concuerda con los hallazgos de diversos investigadores en los diferentes niveles educativos en el mundo. La influencia del nivel de estudios de la madre se debe en gran parte a que es la que participa más activamente en actividades escolares y quien coadyuva al desarrollo de habilidades cognitivas en sus hijos debido a la convivencia, ya que, a pesar del cambio social, son las madres las que se encuentran más relacionadas con la educación de los hijos.

Los resultados permitieron comprobar que a nivel universitario el rendimiento académico de los estudiantes está influenciado por la ocupación del padre, debido a que aquellos que se tienen ocupaciones con mejores ingresos pueden proporcionar fuentes y recursos físicos y electrónicos para el aprendizaje y así mejorar sus habilidades cognitivas.

Los descubrimientos en la presente investigación permiten plantear que las universidades contemplen como beneficiarios de sus becas a estudiantes de bajos recursos, pero más allá de una forma meramente asistencialista: deberían tomar a cursos de regularización para poder acceder a la beca. De esta forma no solo se estaría incrementando sus posibilidades económicas para acceder a recursos y fuentes de aprendizaje, también se estarían mejorando sus habilidades cognitivas.

El incremento en el rendimiento académico de los estudiantes podrá contribuir a la disminución del abandono escolar y en el largo plazo a la mejora de la calidad de vida de la población. 


\section{Referencias}

Abuya, B., Mumah, J., Austrian, K., Mutisya, M. and Kabiru, C. (2018). Mothers' Education and Girls' Achievement in Kibera: The Link With Self-Efficacy. SAGE Open, $8(1)$.

Albor, R., Dau , M. y Ruíz, J. (2014). Calidad institucional y rendimiento académico. El caso de las universidades del Caribe colombiano. Perfiles educativos, 36(143), 10-29.

Baumann, C. and Hamin. (2011). The role of culture, competitiveness and economic performance in explaining academic performance: a global market analysis for international student segmentation. Journal of Marketing for Higher Education, 21(2), 181-201.

Canales, A. (2016). Diferencias socioeconómicas en la postulación a las universidades chilenas: el rol de factores académicos y no académicos. Calidad en la educación, (44), 129-157.

Casanova, J., Cervero, A., Núñez, J., Almeida, L. and Bernardo, A. (2018). Factors that determine the persistence and dropout of university students. Psicothema, 30(4), 408-414.

Chaparro, A., González, C. y Caso, J. (2016). Familia y rendimiento académico: configuración de perfiles estudiantiles en secundaria. Revista Electrónica de Investigación Educativa, 18(1), 53-68.

Chi, S., Wang, Z., Liu, X. and Zhu, L. (2017). Associations among attitudes, perceived difficulty of learning science, gender, parents' occupation and students' scientific competencies. International Journal of Science Education, 39(16), 2171-2188.

Coschiza, C. C., Fernández, J. M., Redcozub, G. G., Nievas, M. y Ruiz, H. (2016). Características Socioeconómicas y Rendimiento Académico. El Caso de una Universidad Argentina. Revista Iberoamericana sobre Calidad, Eficacia y Cambio en Educación, 14(3), 51-76.

Cuenca, A. (2016). Desigualdad de oportunidades en Colombia: impacto del origen social sobre el desempeño académico y los ingresos de graduados universitarios. Estudios Pedagógicos, 42(2), 69-93.

De Coninck, D., Matthijs, K. and Luyten, P. (2019). Subjective well-being among firstyear university students: A two-wave prospective study in Flanders, Belgium. Student Success, 10(1), 33-45. 


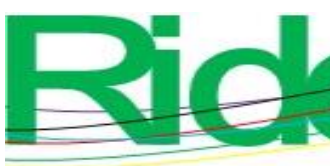

Revista Iberoamericana para la Investigación y el Desarrollo Educativo ISSN $2007-7467$

Díaz, D. y Morales, M. (2011). La reciprocidad en la parentalidad y rendimiento académico en adolescentes. Uaricha. Revista de Psicología, 8(16), 25-35.

Erola, J., Jalonen, S. and Lehti, H. (2016). Parental education, class and income over early life course and children's achievement. Research in Social Stratification and Mobility, 44, 33-43.

Escobar, M. (1992). El análisis de segmentación: conceptos y aplicaciones. Madrid, España: Instituto Juan March de Estudios e Investigaciones en Madrid.

García, A., Soto, L. y Ochoa, R. (2013). Papá y mamá: dos maneras de ver la vida... y una misma educación. Enseñanza e Investigación en Psicología, 18(1), 51-67.

Harding, J., Morris, P. and Hughes, D. (2015). The Relationship Between Maternal Education and Children's Academic Outcomes: A Theoretical Framework. Journal of Marriage and Family, 77(1), 60-76.

Kim, S. (2018). How and why fathers are involved in their children's education: gendered model of parent involvement. Educational Review, 70(3), 280-299.

Koshy, P., Dockery, A. M. and Seymour, R. (2019). Parental expectations for young people's participation in higher education in Australia. Studies in Higher Education, 44(2), 302-317.

Mamiseishvili, K. (2012). International student persistence in U.S. postsecondary institutions. Higher Education, 64(1), 1-17.

Manchón, L. C. y Cordero, F. J. (2014). Factores explicativos del rendimiento en educación primaria: un análisis a partir de TIMSS 2011. Estudios sobre educación, 27, 9-35.

Marks, G. N. (2011). Issues in the Conceptualisation and Measurement of Socioeconomic Background: Do Different Measures Generate Different Conclusions? Social Indicators Research, 104(2), 225-251.

Muelle, L. (2016). Factores de riesgo en el bajo desempeño académico y desigualdad social en el Perú según PISA 2012. Apuntes, 43 (79), 9-45.

Ravelo, E. (2013). Descripción de factores sociodemográficos y socioafectivos y su relación con el desempeño académico de los estudiantes de cuarto semestre de psicología de una institución de educación superior. Psicogente, 16(29), 13-31.

Reyes, R., Godínez, F., Ariza, F. J., Sánchez, F. y Torreblanca, O. F. (2014). Un modelo empírico para explicar el desempeño académico de estudiantes de bachillerato. Perfiles Educativos, 36(146), 45-62.

Rodríguez, G. (2007). Generalized Linear Models. Princeton University. Recuperado de https://data.princeton.edu/wws509/notes/. 
Rodríguez, R. D. y Guzmán, R. R. (2019). Rendimiento académico de adolescentes declarados en situación de riesgo. Revista de Investigación Educativa, 37(1), 147162.

Simões, C., Rivera, F., Moreno, C. and Gaspar de Matos, M. (2018). School Performance Paths: Personal and Contextual Factors Related to Top Performers and Low Achievers in Portugal and Spain. The Spanish Journal of Psychology, 21(e36), 115.

Takeda, T. and Lamichhane, K. (2018). Determinants of schooling and academic achievements: Comparison between children with and without disabilities in India. International Journal of Educational Development, 61, 184-195.

Waterman, E. and Lefkowitz, E. (2017). Are mothers' and fathers' parenting characteristics associated with emerging adults' academic engagement? Journal of family issues, 38(9), 1239-1261.

Yáñez, A. I., Vera, J. Á. y Mungarro, J. E. (2014). El proceso de admisión de las escuelas normales y los antecedentes socioeconómicos como predictores del rendimiento académico. Revista Intercontinental de Psicología y Educación, 16(2), 111-129.

\begin{tabular}{|l|l|}
\hline Rol de Contribución & Autor (es) \\
\hline Conceptualización & Martha Jiménez García y María Vianey Espejel García (igual) \\
\hline
\end{tabular}


Revista Iberoamericana para la

Investigación y el Desarrollo Educativo

ISSN $2007-7467$

\begin{tabular}{|c|c|}
\hline Metodología & María Vianey Espejel García \\
\hline Software & María Vianey Espejel García \\
\hline Validación & María Vianey Espejel García \\
\hline Análisis Formal & María Vianey Espejel García \\
\hline Investigación & María Vianey Espejel García (principal) y Martha Jiménez García (que apoya) \\
\hline Recursos & Martha Jiménez García y María Vianey Espejel García (igual) \\
\hline Curación de datos & María Vianey Espejel García \\
\hline $\begin{array}{l}\text { Escritura - Preparación del borrador } \\
\text { original }\end{array}$ & María Vianey Espejel García \\
\hline Escritura - Revisión y edición & María Vianey Espejel García \\
\hline Visualización & María Vianey Espejel García \\
\hline Supervisión & María Vianey Espejel García y Martha Jiménez García (igual) \\
\hline Administración de Proyectos & María Vianey Espejel García \\
\hline Adquisición de fondos & Martha Jiménez García \\
\hline
\end{tabular}

